\title{
Interplay between Charge and Magnetic Orderings in the Zero-Bandwidth Limit of the Extended Hubbard Model for Strong On-Site Repulsion
}

\author{
K. KapCiA*, W. KŁobus and S. RobaszKiewiCZ
}

Faculty of Physics, Adam Mickiewicz University, Umultowska 85, 61-614 Poznań, Poland

\begin{abstract}
A simple effective model of charge ordered and (or) magnetically ordered insulators is studied. The tight binding Hamiltonian analyzed consists of (i) the effective on-site interaction $U$, (ii) the intersite density-density interaction $W$ and (iii) intersite magnetic exchange interaction $J^{z}$ (or $J^{x y}$ ) between nearest-neighbors. The intersite interaction are treated within the mean-field approximation. One shows that the systems considered can exhibit very interesting multicritical behaviors, including among others bicritical, tricritical, tetracritical and critical end points. The analysis of the model has been performed for an arbitrary electron concentration as well as an arbitrary chemical potential in the limit of strong on-site repulsion $(U \rightarrow+\infty)$. The phase diagrams obtained in such a case are shown to consist of at least 9 different states, including four homogeneous phases: nonordered (NO), ferromagnetic $(\mathrm{F})$, charge ordered $(\mathrm{CO})$, ferrimagnetic (intermediate, I) and five types of phase separation: NO-NO, F-NO, F-F, CO-F, CO-I.
\end{abstract}

PACS: 71.10.Fd, 71.45.Lr, 75.30.Fv, 64.75.Gh, 71.10.Hf

\section{Introduction}

Electron charge orderings and their interplay with magnetism are relevant to broad range of important materials such as manganites, multiferroics and other strongly correlated electron systems [1-3]. In this report we discuss an effective model of charge ordered and (or) magnetically ordered insulators.

We consider the following model Hamiltonian of a fermion lattice system in the atomic limit:

$$
\begin{aligned}
\hat{H} & =U \sum_{i} \hat{n}_{i \uparrow} \hat{n}_{i \downarrow}-\mu \sum_{i} \hat{n}_{i} \\
& +\frac{W}{2} \sum_{\langle i, j\rangle} \hat{n}_{i} \hat{n}_{j}-2 J \sum_{\langle i, j\rangle} \hat{s}_{i}^{z} \hat{s}_{j}^{z},
\end{aligned}
$$

where $\hat{c}_{i \sigma}^{+}$denotes the creation operator of an electron with spin $\sigma$ at the site $i, \hat{n}_{i \sigma}=\hat{c}_{i \sigma}^{+} \hat{c}_{i \sigma}, \hat{n}_{i}=\sum_{\sigma} \hat{n}_{i \sigma}$, $\hat{s}_{i}^{z}=(1 / 2)\left(\hat{n}_{i \uparrow}-\hat{n}_{i \downarrow}\right)$. $U, W$, and $J$ are the interaction parameters (on-site and intersite between the nearest neighbors). $\mu$ is the chemical potential, depending on the concentration of electrons

$$
n=\frac{1}{N} \sum_{i}\left\langle\hat{n}_{i}\right\rangle,
$$

with $n \in[0,2], N$ is the total number of lattice sites. Our denotations: $n_{Q}=\frac{1}{2}\left(n_{\mathrm{A}}-n_{\mathrm{B}}\right), n_{\alpha}=\frac{2}{N} \sum_{i \in \alpha}\left\langle\hat{n}_{i}\right\rangle$,

\footnotetext{
* corresponding author; e-mail: kakonrad@amu.edu.pl
}

$m=\frac{1}{N} \sum_{i}\left\langle\hat{s}_{i}^{z}\right\rangle, m_{Q}=\frac{1}{2}\left(m_{\mathrm{A}}-m_{\mathrm{B}}\right), m_{\alpha}=\frac{2}{N} \sum_{i \in \alpha}\left\langle\hat{s}_{i}^{z}\right\rangle$, and $\alpha=\mathrm{A}, \mathrm{B}$ labels the sublattices. $W_{0}=z W$ and $J_{0}=z J$, where $z$ is the number of the nearest neighbors. Only the two-sublattice orderings on the alternate lattices are considered in this report.

The interactions $U, W$, and $J$ will be treated as effective ones and be assumed to include all the possible contributions and renormalizations. One should notice that in the absence of external magnetic field the ferromagnetic $(J>0)$ interactions are simply mapped onto the antiferromagnetic ones $(J<0)$ by redefining the spin direction in one sublattice in lattices decomposed into two interpenetrating sublattices. Thus, we restrict ourselves to the case $J>0$.

In the analysis we have adopted a variational approach (VA), which treats the on-site interaction term $(U)$ exactly and the intersite interactions $(W, J)$ within the mean-field approximation (MFA).

For model (1) a ground state phase diagram as a function of $\mu$ [4] (exact results) and special cases at $T \geq 0$ (in VA) such as $W=0$ [5], $J=0[6-8]$ and $n=1$ [9] have been investigated till now.

Within the VA the intersite interactions are decoupled within the MFA, which allows us to find a free energy per site $f(n)$. One can also calculate the averages: $n$, $n_{Q}, m$ and $m_{Q}$, which gives a set of four self-consistent equations (for homogeneous phases). This set for $T \geq 0$ is solved numerically and one obtains $n_{Q}, m, m_{Q}$ and $n$ (or $\mu$ ) when $\mu$ (or $n$ ) is fixed. It is important to find a solution corresponding to the lowest energy. 
TABLE

Definitions of homogeneous phases and PS states.

\begin{tabular}{c|c|c|c|c}
\hline \hline Phase & Order parameters & State & $n_{ \pm}$ & $n_{\mp}$ \\
\hline & & PS2 & CO & NO \\
$\mathrm{CO}$ & $n_{Q} \neq 0, m=0, m_{Q}=0$ & PS3 & NO & NO \\
$\mathrm{F}$ & $n_{Q}=0, m \neq 0, m_{Q}=0$ & PS4 & $\mathrm{F}$ & $\mathrm{NO}$ \\
$\mathrm{I}$ & $n_{Q} \neq 0, m \neq 0, m_{Q} \neq 0$ & $\mathrm{PS} 5$ & $\mathrm{I}$ & $\mathrm{CO}$ \\
$\mathrm{NO}$ & $n_{Q}=0, m=0, m_{Q}=0$ & $\mathrm{PS} 6$ & $\mathrm{~F}$ & $\mathrm{CO}$ \\
& & $\mathrm{PS} 7$ & $\mathrm{~F}$ & $\mathrm{~F}$
\end{tabular}

The phase separation (PS) is a state in which two domains with different electron concentrations exist (coexistence of two homogeneous phases). The energy of PS states is given by

$$
f_{\mathrm{PS}}\left(n_{+}, n_{-}\right)=k f_{+}\left(n_{+}\right)+(1-k) f_{-}\left(n_{-}\right),
$$

where $E_{ \pm}\left(n_{ \pm}\right)$are values of a free energy at $n_{ \pm}$corresponding to the lowest energy homogeneous solutions and $k=\frac{n-n_{-}}{n_{+}-n_{-}}$is a fraction of the system with density $n_{+}$.

In the report we have used the following convention. A second (first) order transition is a transition between homogeneous phases with a (dis-)continuous change of the order parameter at the transition temperature. A transition between homogeneous phase and PS state is symbolically named as a "third order" transition. At this transition a size of one domain in the PS state decreases continuously to zero at the transition temperature. We have also distinguished a second order transition between two PS states, at which a continuous change of the order parameter in both domains takes place.

In this report we present the VA results for model (1) in the limit $U \rightarrow+\infty$. This case corresponds to the subspace excluding the double occupancy of sites (by electrons for $n<1$ or holes for $n>1$ ). The obtained phase diagrams (for fixed $n$ ) are symmetric with respect to half-filling $(n=1)$ because of the particle-hole symmetry of Hamiltonian (1). The phases and states, which can exist on the phase diagrams of model considered, are collected in Table.

\section{Results and discussion for $U \rightarrow+\infty$}

In this section we discuss the behavior of the system in the limit of infinite on-site interaction $(U \rightarrow+\infty)$. The ground state diagrams obtained in this limit are shown in Fig. 1. The energies of a few states are degenerated if the system is considered for fixed $n$. However, at finite temperatures the degeneration is removed. LCO is the particular $\mathrm{CO}$ phase with $n_{Q}=0.5$ and $n=0.5$ or $n=1.5$.

The selected finite temperature phase diagrams, obtained for fixed $\mu$ as well as for fixed $n$, are presented in Figs. 2-5. One should notice that the first order transitions on the diagrams for fixed $\mu$ corresponds to the "third order" transitions and occurrence of PS states on the diagrams for fixed $n$. The second order transition $\mathrm{F}-\mathrm{NO}$ occurs at $k_{\mathrm{B}} T=J_{0}|1-n|$ independently of $W / J$.
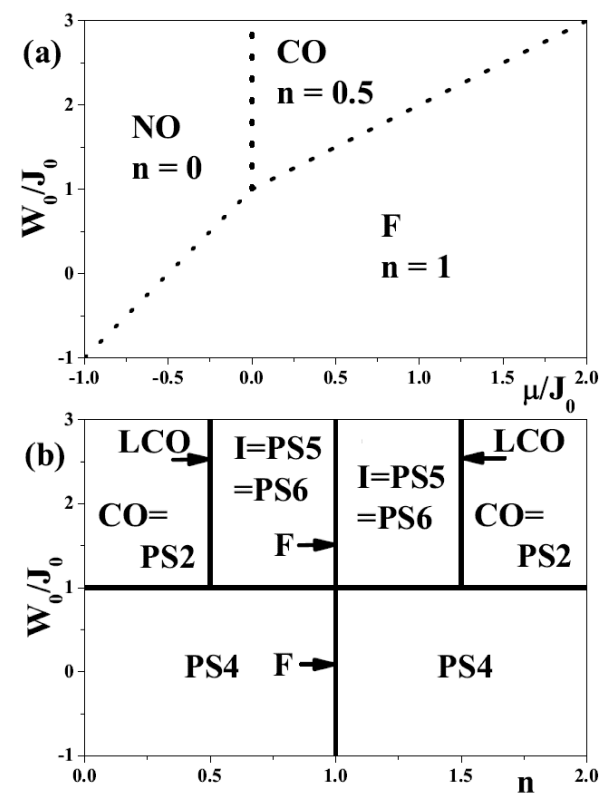

Fig. 1. Ground state phase diagrams in the $U \rightarrow+\infty$ limit: (a) as a function of $\mu / J_{0}$ and (b) as a function of $n$. Dotted lines denote discontinuous transitions (on the part (a)).

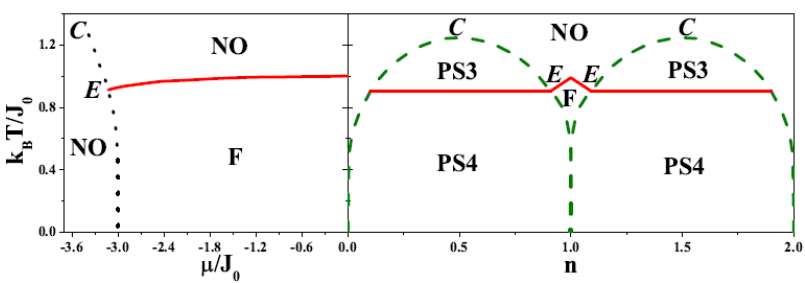

Fig. 2. Finite temperature phase diagrams for $W / J=-5$ (the $U \rightarrow+\infty$ limit). Dotted, solid and dashed lines indicate first order, second order and "third order" boundaries, respectively. Details in text.

The labels of the critical points for phase separation are given corresponding to those in [10], where the BlumeEmery-Griffiths model was considered. Critical points indicated on the phase diagrams (descriptions in correspondence to diagrams as a function of $\mu / J_{0}$, Figs. 2-5): (i) $T$ - tricritical point: change of the transition order,

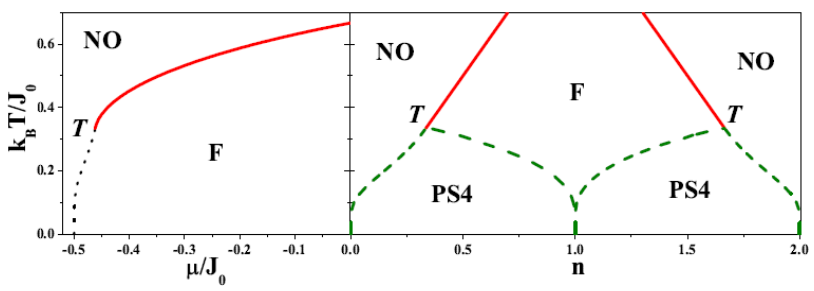

Fig. 3. Finite temperature phase diagrams for $W / J=0$ and $U \rightarrow+\infty$. Denotations as in Fig. 2 . 


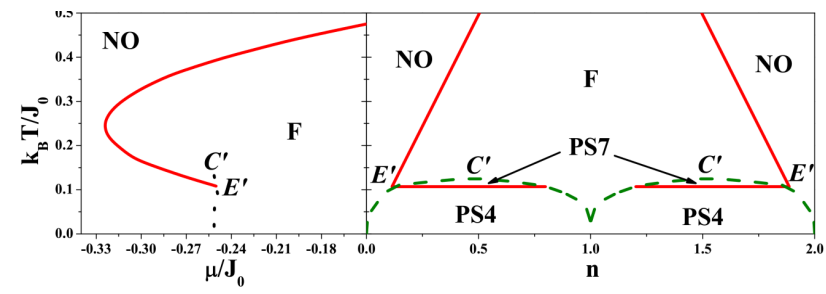

Fig. 4. Finite temperature phase diagrams for $W / J=0.5$ and $U \rightarrow+\infty$. Denotations as in Fig. 2 .

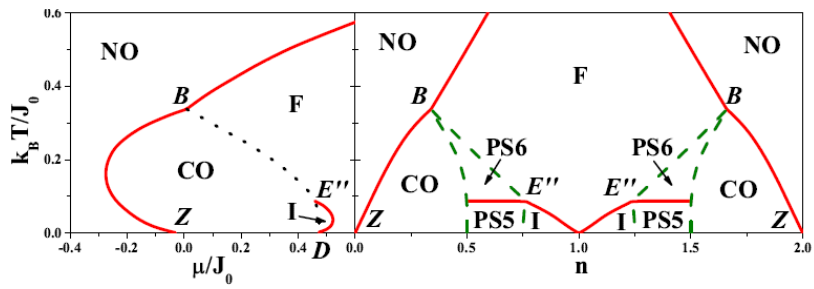

Fig. 5. Finite temperature phase diagrams for $W / J=1.5$ and $U \rightarrow+\infty$. Denotations as in Fig. 2 .

(ii) $C, C^{\prime}$ - isolated critical (bicritical end) points: the end of 1st order transition line, (iii) $E, E^{\prime}, E^{\prime \prime}$ - critical end points: connection of two 1st order lines and one 2nd order line, (iv) $Z$ - zero-temperature critical point: the end of 2 nd order transition line at $T=0$, (v) $D$ - zero-temperature highly degenerate point: the end of both 1st and 2nd order transitions lines at $T=0$, (vi) $B-$ bicritical point: connection of one 1 st order and two 2 nd order lines.

For large negative values of $W / J$, the second order F-NO line terminates at $E$-point on a first order line, which itself ends at $C$-point (Fig. 2). For values of $W / J \approx 0$ (Fig. 3), the transition lines meet $T$-point, which is connected with a change of $\mathrm{F}-\mathrm{NO}$ transition order [5].

With increasing $W / J$ the tricritical behavior changes into another one with the $C^{\prime}$-point located inside the $\mathrm{F}$ phase (cf. Fig. 4, for $W / J=0.5$ ).

For $W / J>1$ (Fig. 5) two new ordered phases appear on the phase diagrams: charge ordered (CO) phase $\left(n_{Q} \neq 0\right)$ and intermediate (I) homogeneous phase, where magnetic order and charge order coexist $\left(n_{Q} \neq 0, m \neq 0\right.$ and $\left.m_{Q} \neq 0\right)$. If $W / J=1.5$ the $\mathrm{F}$ and $\mathrm{CO}$ phases are separated from the NO phase by two second order lines that meet at $B$-point. The transitions between $\mathrm{CO}$ and phases with $m \neq 0(\mathrm{~F}, \mathrm{I})$ are first order. The $\mathrm{F}$ and I phases are separated by second order line ending at $E^{\prime \prime}$-point.

\section{Final remarks}

It is seen that a large variety of phase transition phenomena are present in the simple model incorporating both magnetic and density degrees of freedom. Derived results are exact in the limit of infinite dimensions, where the MFA treatment of intersite interactions becomes the rigorous one.
Our model in the limit $U \rightarrow+\infty$ is equivalent to the Blume-Emery-Griffiths model, which is the general spin $S=1$ Ising model with nearest-neighbor interactions and up-down symmetry. Thus obtained diagrams have the similar structure as those presented in Ref. [10].

One notices that in the $U \rightarrow-\infty$ limit of model (1) the magnetic orderings are completely suppressed and the CO phase (for $W>0$ ) [6-9] or the PS: NO-NO state (for $W<0$ ) [11] can occur. For $W=0$ only the NO phase is stable at any $k_{\mathrm{B}} T \geq 0$ in this limit.

Let us point out that in the MFA the following equivalence occurs: $2 J \sum_{\langle i, j\rangle} \hat{s}_{i}^{z} \hat{s}_{j}^{z} \stackrel{\text { MFA }}{\longleftrightarrow} J \sum_{\langle i, j\rangle}\left(\hat{s}_{i}^{+} \hat{s}_{j}^{-}+\hat{s}_{j}^{+} \hat{s}_{i}^{-}\right)$. In both cases the self-consistent equations have the same form and a magnetization along the $z$-axis becomes a magnetization in the $x y$-plane [12].

\section{Acknowledgments}

The work (K.K., S.R.) has been financed by National Science Center (NCN) as a research project in years 2011-2013, grant No. DEC-2011/01/N/ST3/00413. K.K. would like to thank the European Commission and Ministry of Science and Higher Education (Poland) for the partial financial support from European Social Fund - Operational Programme "Human Capital" POKL.04.01.01-00-133/09-00 — "Pro-innovation training, competent staff, graduates of the future".

\section{References}

[1] R. Micnas, J. Ranninger, S. Robaszkiewicz, Rev. Mod. Phys. 62, 113 (1990).

[2] T. Goto, B. Lüthi, Adv. Phys. 52, 67 (2003); E. Dagotto, T. Hotta, A. Moreo, Phys. Rep. 344, 1 (2001).

[3] J. van den Brink, D.I. Khomskii, J. Phys., Condens. Matter 20, 434217 (2008).

[4] U. Brandt, J. Stolze, Z. Phys. B 62, 433 (1986); J. Jędrzejewski, Physica A 205, 702 (1994).

[5] W. Kłobus, K. Kapcia, S. Robaszkiewicz, Acta Phys. Pol. A 118, 353 (2010).

[6] R. Micnas, S. Robaszkiewicz, K.A. Chao, Phys. Rev. B 29, 2784 (1984).

[7] K. Kapcia, W. Kłobus, S. Robaszkiewicz, Acta Phys. Pol. A 118, 350 (2010).

[8] K. Kapcia, S. Robaszkiewicz, J. Phys., Condens. Matter 23, 105601 (2011); 23, 249802 (2011).

[9] S. Robaszkiewicz, Acta Phys. Pol. A 55, 453 (1979); Phys. Status Solidi B 70, K51 (1975).

[10] W. Hoston, A.N. Berker, Phys. Rev. Lett. 67, 1027 (1991); C. Ekiz, M. Keskin, Phys. Rev. B 66, 054105 (2002).

[11] R.J. Bursill, C.J. Thompson, J. Phys. A: Math. Gen. 26, 4497 (1993); F. Macini, F.P. Mancini, Eur. Phys. J. B 73, 581 (2010).

[12] W. Kłobus, M.Sc. Thesis, Adam Mickiewicz University, Poznań 2009. 